\title{
Catabolism of L-Lysine by Pseudomonas aeruginosa
}

\author{
By J. C. FOTHERGILL AND J. R. GUEST \\ Department of Microbiology, University of Sheffield, Sheffield, SIo $2 T N$
}

(Received I October 1976)

\begin{abstract}
SUMMARY
Pseudomonas aeruginosa PACI grows poorly on L-lysine as sole source of carbon but mutant derivatives which grow rapidly were readily isolated. Studies with one such mutant, $\boldsymbol{P}$. aeruginosa PAC586, supported the existence of a route for L-lysine catabolism which differs from those reported previously in other species of Pseudomonas. The postulated route, the cadaverine or decarboxylase pathway, is initiated by the decarboxylation of L-lysine and involves the following steps: L-lysine $\rightarrow$ cadaverine $\rightarrow$ I-piperideine $\rightarrow$-aminovalerate $\rightarrow$ glutarate semialdehyde $\rightarrow$ glutarate. Evidence for this pathway is based on the characterization of the pathway reactions and the induction of the corresponding enzymes by growth on Llysine. The first three enzymes were also induced by growth on cadaverine and to a lesser extent by 5-aminovalerate. No evidence was obtained for the presence of pathways involving L-lysine 2-monooxygenase or L-pipecolate dehydrogenase, but another potential route for L-lysine catabolism initiated by L-lysine 6-aminotransferase was detected. Studies with mutants unable to grow on L-lysine supported the existence of more than one catabolic pathway for L-lysine in this organism and indicated that all routes converge on a pathway for glutarate catabolism which generates acetyl-CoA. Pipecolate catabolism also appeared to converge on the glutarate pathway in $P$. aeruginosa. The results suggested that the growth rate of the parental strain is limited by the rate of transport and/or decarboxylation of L-lysine. The cadaverine pathway was present, but not so highly induced, in the parental strain $P$. aeruginosa PACI. Pseudomonas fluorescens contained enzymes of both the cadaverine (decarboxylase) and oxygenase pathways, strains of $P$. putida (biotypes A and B) contained enzymes of the oxygenase pathway but not the decarboxylase pathway and $P$. multivorans appeared deficient in both. All these species possessed L-lysine aminotransferase activity.
\end{abstract}

\section{INTRODUCTION}

Three pathways for lysine catabolism by aerobic bacteria are outlined in Fig. I. These are the 5-aminovalerate (or oxygenase) pathway, the pipecolate pathway and a transaminase pathway.

Evidence for the 5-aminovalerate pathway is based on the characterization of the individual enzyme-catalysed reactions in Pseudomonas fluorescens and Pseudomonas putida and also the induction of some of the enzymes by lysine (Reitz \& Rodwell, 1970; Vandecasteele \& Hermann, 1972). Likewise, the pipecolate pathway, containing cyclic intermediates, was defined initially by Rodwell and coworkers in P. putida P2, a strain isolated on pipecolate. The relevance of pipecolate to lysine catabolism was indicated by the finding that pipecolate-grown organisms were induced for lysine degradation and that L-lysine induced the synthesis of pipecolate dehydrogenase (Baginsky \& Rodwell, 1966) and I-piperideine-2carboxylate reductase (Chang \& Adams, 1974). Although the two pathways are connected by lysine racemase, it is now clear from isotopic labelling studies and by inhibition of the 


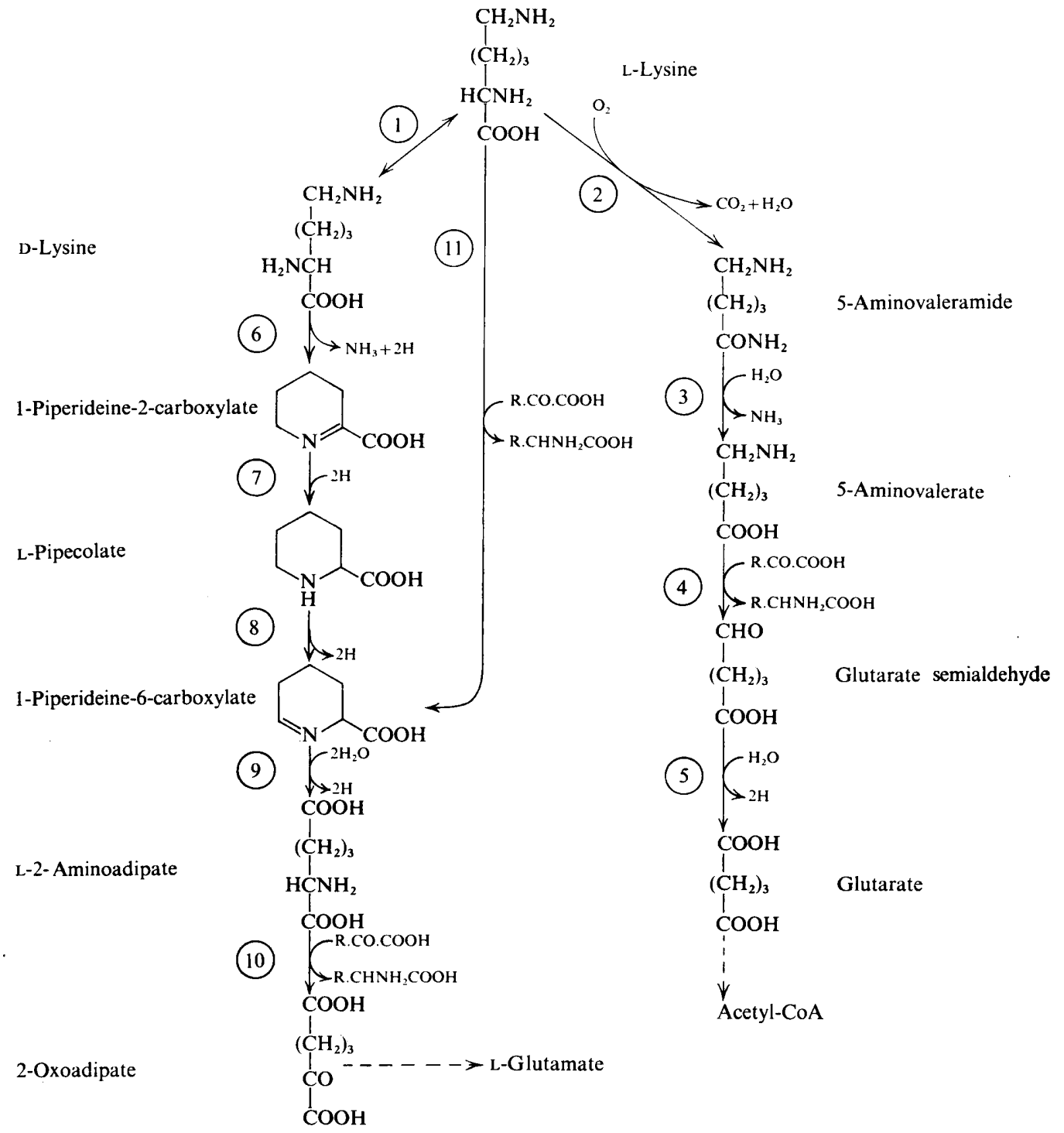

Fig. I. Scheme showing the initial steps in lysine catabolism by aerobic bacteria. The enzymes and corresponding references are: (I) lysine racemase (EC. 5.I.I.5), Ichihara, Furiya \& Suda (1960); (2) L-lysine 2-monooxygenase (EC. I.13.12.2), Takeda et al. (1969); (3) 5-aminovaleramide amidase (EC. 3.5.1.-), Reitz \& Rodwell (1970); (4) 5-aminovalerate aminotransferase (EC. 2.6.1 .-), Ichihara, Ichihara \& Suda (1960); (5) glutarate-semialdehyde dehydrogenase (EC. I.2.I.20), Ichihara \& Ichihara (196I); (6) postulated reaction; (7) I-piperideine-2-carboxylate reductase (EC. I . 5 . I .-), Chang \& Adams (1974); (8) L-pipecolate dehydrogenase (EC. I . 5 . 99 . 3), Baginsky \& Rodwell (1966); (9) I-piperideine-6-carboxylate dehydrogenase (EC. 1 . 5 . I .-), Calvert \& Rodwell (I966); (IO) L-2-aminoadipate aminotransferase (EC. 2.6. I . 39), Hartline \& Rodwell (197I); (I I) L-lysine 6-aminotransferase (EC. 2.6. I .36), Soda, Misono \& Yamamoto (1968). Reactions 2 to 5 represent the 5 -aminovalerate (or oxygenase) pathway, reactions 6 to Io represent the pipecolate pathway and reaction II may be considered as a third route, the transaminase pathway. The cyclic compounds I-piperideine-2-carboxylate and I-piperideine-6-carboxylate are the dehydrated cyclic forms of 2-oxo-6-aminocaproic acid and 2-aminoadipate 5-semialdehyde, respectively. 
racemase that degradation of $L$-lysine is primarily via 5 -aminovalerate and degradation of D-lysine occurs via pipecolate, in P. putida P2 (Miller \& Rodwell, I97I $a$ ). Furthermore, in another strain of $P$. putida biotype A (ATCCI5070), studies with mutants blocked in the 5aminovalerate pathway showed this route to be obligatory for growth on L-lysine (Chang \& Adams, 1974). Growth of the mutants on D-lysine was unaffected, and it was concluded that although there is sufficient racemase activity to permit cross-induction of the pipecolate pathway by L-lysine (and the 5 -aminovalerate pathway by D-lysine) it fails to support growth.

Transamination of L-lysine to form I-piperideine-6-carboxylate has been reported in Llysine-grown Achromobacter liquidum and Flavobacterium species (Soda \& Misono, I968; Soda, Misono \& Yamamoto, I968) and this provides a third route for lysine catabolism.

Glutarate, the product of the 5-aminovalerate pathway, is probably metabolized to acetyl-CoA via glutaryl-CoA, glutaconyl-CoA, 3-hydroxybutyryl-CoA and acetoacetyl$\mathrm{CoA}$, as described in $\boldsymbol{P}$. fluorescens (Numa et al., I964). In contrast, the product of the other routes, 2-oxoadipate, appears to be metabolized via 2-hydroxyglutarate to 2-oxoglutarate and glutamate (Reitz \& Rodwell, I969; Hartline \& Rodwell, I97I). It is not clear whether these compounds are of necessity the ultimate products because decarboxylation of any of the $\mathrm{C}_{6}$ intermediates would yield products which would converge on glutarate.

Studies with Pseudomonas aeruginosa, originally intended to investigate the biochemical control and genetic organization of lysine catabolism and other converging pathways, have provided evidence for another pathway of L-lysine degradation. This pathway, the cadaverine or decarboxylase pathway, is initiated by decarboxylation and provides an alternative route to 5 -aminovalerate and eventually glutarate.

\section{METHODS}

Bacterial strains. Pseudomonas aeruginosa PACI (NCIBI0848) was a gift from Professor P. H. Clarke as was the isocitrate lyase mutant, PAC50I, originally designated AtI (Skinner \& Clarke, 1968). Mutant PAC586 was derived from PACI by selection for faster growth with L-lysine as sole source of carbon. Other cultures and their sources were: $P$. aeruginosa PAOI (ATCCI7503), Dr M. B. Kemp; P. putida biotype B (ATCCI7472), Professor R. Y. Stanier; $P$. fluorescens (ATCCII250), $P$. putida biotype A (ATCCI2633) and $P$. multivorans (ATCC17759), the American Type Culture Collection (Stanier, Palleroni \& Doudoroff, 1966). Stock cultures of $P$. aeruginosa PAC586 were maintained on plates of L-lysine minimal medium subcultured at monthly intervals, incubated for $30 \mathrm{~h}$ and stored at $2{ }^{\circ} \mathrm{C}$. Other stocks were maintained on plates of nutrient agar and subcultured and stored in the same way during routine use. Stocks of each culture were also maintained on slopes of nutrient agar at $2{ }^{\circ} \mathrm{C}$ under paraffin for long-term storage. Strains of $P$. aeruginosa were cultured at $37^{\circ} \mathrm{C}$, but $30^{\circ} \mathrm{C}$ was used for all other species.

Isolation of mutants. Spontaneous mutants $\left(\mathrm{Lut}^{+}\right)$of PAOI and PACI capable of faster growth on L-lysine as sole carbon source were isolated by direct selection of the larger colonies which appeared after plates of L-lysine minimal medium were spread with $10^{9}$ organisms. They were also selected by repeated subculture in lysine minimal medium. Five subcultures comprising a total of approximately 45 generations were used although no increase in growth rate was detected after 25 generations.

Mutants of $P$. aeruginosa PAC586 able to grow with succinate but not L-lysine were selected by treating exponential nutrient broth cultures with I-methyl-3-nitro-I-nitrosoguanidine $\left(100 \mu \mathrm{g} \mathrm{ml}^{-1}\right.$ ) for $40 \mathrm{~min}$ at $37^{\circ} \mathrm{C}$ in $0 . \mathrm{I} \mathrm{M}$-sodium citrate buffer $\mathrm{pH} 5.4$. This was followed 
by three cycles of mutant enrichment consisting of expression in succinate minimal medium and exposure of early-exponential phase cultures in lysine minimal medium to antibiotic for $18 \mathrm{~h}$. The antibiotic concentrations were: Ist cycle, carbenicillin $\left(200 \mu \mathrm{g} \mathrm{ml}^{-1}\right)$; 2nd cycle, carbenicillin $\left(500 \mu \mathrm{g} \mathrm{ml}^{-1}\right) ; 3$ rd cycle, carbenicillin $\left(500 \mu \mathrm{g} \mathrm{ml}^{-1}\right)$ plus D-cycloserine (I00 $\mu \mathrm{g} \mathrm{ml}^{-1}$ ). After each cycle, survivors were collected by centrifuging, resuspended in phosphate buffer $(0.05 \mathrm{M}, \mathrm{pH} 7 \cdot 0)$ and plated on succinate minimal medium. Mutants were then detected by replica-plating to lysine minimal medium; approximately $17 \%$ and $90 \%$ of the survivors had the desired phenotype after the second and third cycles, respectively.

Media. The nutrient broth used routinely contained $\left(\mathrm{g}^{-1}\right)$ : Bacto nutrient broth (Difco), 8; Bacto yeast extract (Difco), 5; and $\mathrm{NaCl}, 8 \cdot 5$. The nutrient agar contained $\left(\mathrm{g}^{-1}\right)$ : Bacto yeast extract (Difco), I0; and Ionagar no. 2 (Oxoid), 10.

The basal minimal medium contained $\left(\mathrm{l}^{-1}\right): \mathrm{NaH}_{2} \mathrm{PO}_{4} .2 \mathrm{H}_{2} \mathrm{O}, 6.0 \mathrm{~g} ; \mathrm{K}_{2} \mathrm{HPO}_{4}$, $10.7 \mathrm{~g}$; $\left(\mathrm{NH}_{4}\right)_{2} \mathrm{SO}_{4}, \mathrm{I} \cdot 0 \mathrm{~g}$; and $5 \mathrm{ml}$ trace element solution. The trace element solution contained $\left(1^{-1}\right): \mathrm{FeSO}_{4} \cdot 7 \mathrm{H}_{2} \mathrm{O}, 82 \mathrm{mg} ; \mathrm{H}_{3} \mathrm{BO}_{3}, 232 \mathrm{mg} ; \mathrm{CoSO}_{4} \cdot 7 \mathrm{H}_{2} \mathrm{O}, 96 \mathrm{mg} ; \mathrm{CuSO}_{4} \cdot 5 \mathrm{H}_{2} \mathrm{O}, 8 \mathrm{mg}$; $\mathrm{MnSO}_{4} \cdot 4 \mathrm{H}_{2} \mathrm{O}, 8 \mathrm{mg} ; \mathrm{Na}_{2} \mathrm{MoO}_{4} \cdot 2 \mathrm{H}_{2} \mathrm{O}, 30 \mathrm{mg} ; \mathrm{ZnSO}_{4} \cdot 7 \mathrm{H}_{2} \mathrm{O}, 174 \mathrm{mg}$; and $\mathrm{MgSO}_{4} \cdot 7 \mathrm{H}_{2} \mathrm{O}$, $20 \mathrm{~g}$. Carbon sources were neutralized, sterilized and added to the sterilized basal medium to give final concentrations of $20 \mathrm{~mm}$ (or $40 \mathrm{~mm}$ for succinate and acetate). Solid media contained Ionagar no. 2 (Oxoid) at $12 \mathrm{~g} \mathrm{1}^{-1}$.

Growth of cultures. Cultures were grown aerobically at 30 or $37^{\circ} \mathrm{C}$ as $500 \mathrm{ml}$ batches in 21 Erlenmeyer flasks on a rotary shaker. Larger batches of I I 1 were grown in a Microferm Fermentor (New Brunswick Scientific Co.). Cultures were harvested in the exponential phase and washed at least twice using a total volume of $0.05 \mathrm{M}$-phosphate buffer $\mathrm{pH} 8 \cdot 0$ equal to that of the original culture.

Manometric experiments. Oxygen uptake was measured at $37^{\circ} \mathrm{C}$ by conventional Warburg manometric techniques. Washed cultures were resuspended in $0.05 \mathrm{M}$-phosphate buffer pH $7 \cdot 0$ and samples containing $3 \mathrm{mg}$ dry wt organisms and $95 \mu \mathrm{mol}$ phosphate in $2 \cdot 3 \mathrm{ml}$ were added to the flasks. The centre wells contained $0.1 \mathrm{ml} \mathrm{KOH}\left(400 \mathrm{~g} \mathrm{l}^{-1}\right)$ and substrates $(6 \mu \mathrm{mol}$ in $0.6 \mathrm{ml})$ were tipped from the side-arms. Gas uptakes were calculated as $\mu \mathrm{l} \mathrm{O}_{2}$ absorbed (mg dry wt) $)^{-1} h^{-1}$ and corrected for the endogenous values obtained without added substrate.

\section{Preparation of extracts and enzymology}

Ultrasonic extracts were prepared by suspending $\mathrm{I} .5 \mathrm{~g}$ wet wt of bacterial paste in $5 \mathrm{ml}$ $0.05 \mathrm{M}$-phosphate buffer $\mathrm{pH} 8 \cdot 0$ and treating the suspensions for two $90 \mathrm{~s}$ periods in an ultrasonic cell disintegrator (M.S.E., $100 \mathrm{~W}$ ). The suspensions were clarified by centrifuging at $15000 \mathrm{~g}$ for $\mathrm{I} 5 \mathrm{~min}$ at $2{ }^{\circ} \mathrm{C}$. The supernatant fluids were used as crude extracts and their protein concentrations were determined by the method of Lowry et al. (I95I) with bovine serum albumin as the standard. Soluble and particulate fractions were prepared by recentrifuging the crude extracts at $105000 \mathrm{~g}$ for $2.5 \mathrm{~h}$ at $2{ }^{\circ} \mathrm{C}$. The pellet obtained was resuspended in phosphate buffer to the original extract volume to give the particulate fraction and the supernatant fluid was used as the soluble fraction. The protein concentrations of the two fractions were the same, i.e. equal to half the concentration of the crude extract. Enzyme specific activities are based on the protein concentrations of the extracts or fractions used in specific assays and expressed as $\mu \mathrm{mol}$ of substrate transformed or product formed $(\mathrm{mg}$ protein $)^{-1} \mathrm{~h}^{-1}$ at the temperature stated. Enzymes were assayed at their $\mathrm{pH}$ optima and in the region of proportionality between initial reaction velocity and protein concentration, and Michaelis constants were determined by double reciprocal plots (Lineweaver \& Burk, 1934). 
L-Lysine decarboxylase [EC. 4.1.1.18; L-lysine carboxy-lyase] was assayed in crude extracts by following the rate of release of labelled $\mathrm{CO}_{2}$ from $\mathrm{L}-\left[\mathrm{U}-{ }^{14} \mathrm{C}\right]$ lysine. The reaction mixtures contained ( $\mu \mathrm{mol}$ in $0.9 \mathrm{ml}$ final volume): pyridoxal $5^{\prime}$-phosphate, $0.05 ; 2$-mercaptoethanol, I0; sodium potassium phosphate buffer $\mathrm{pH} \mathrm{6.8,} \mathrm{I00;} \mathrm{and} \mathrm{crude} \mathrm{extract}$ equivalent to I to $2 \mathrm{mg}$ protein. Mixtures were pre-incubated for $3 \mathrm{~min}$ at $37^{\circ} \mathrm{C}$. After cooling to $0{ }^{\circ} \mathrm{C}, 12 \mathrm{~cm}^{2}$ glass filter paper wicks impregnated with $0.2 \mathrm{ml}$ hyamine hydroxide ( $\mathrm{I}$ in methanol; scintillation grade; Nuclear Enterprises, Beenham, Berkshire) were suspended above the mixtures. Substrate, $\mathrm{I} \cdot 05 \mu \mathrm{Ci} \mathrm{L}-\left[\mathrm{U}-{ }^{14} \mathrm{C}\right]$ lysine $(20 \mu \mathrm{mol}$ in $0 \cdot \mathrm{I} \mathrm{ml})$, was added, the tubes were sealed immediately and the reactions were started by warming to $37^{\circ} \mathrm{C}$. Reactions were stopped by injecting $0.5 \mathrm{ml} 5 \mathrm{M}-\mathrm{HCl}$ through the gas-proof seal. After allowing $30 \mathrm{~min}$ for $\mathrm{CO}_{2}$ absorption, the wicks were transferred to vials containing $7.5 \mathrm{ml} \mathrm{NE21} 3$ scintillant (Nuclear Enterprises) and counted in Nuclear Chicago 680I or Isocap 300 scintillation counters. Quench corrections were made by the channels ratio technique and the counts (d.p.m.) were related to the specific activity of L-lysine decarboxylase. Anaerobic assays were performed under $\mathrm{N}_{2}\left(\mathrm{O}_{2}\right.$-free) in sealed tubes and lysine was added by syringe. Reactions stopped at zero time and controls containing heated extract or lacking lysine yielded no labelled $\mathrm{CO}_{2}$. A detailed kinetic study showed that the reaction quickly reached a constant rate which was maintained for over $30 \mathrm{~min}$. The early lag was equivalent to $\mathrm{I}$ min and the routine incubation $(\mathrm{I} 6 \mathrm{~min}$ ) corresponded to $15 \mathrm{~min}$ at the rate recorded.

L-Lysine 6-aminotransferase [EC. 2.6.1 .36; L-lysine: 2-oxoglutarate 6-aminotransferase] and cadaverine aminotransferase [EC. 2.6. I .29; diamine:2-oxoglutarate aminotransferase] were assayed by the same method based on those of Soda et al. (1968) and Kim (1964). The reaction mixtures contained ( $\mu \mathrm{mol}$ in $2 \mathrm{ml}$ final volume): 2-oxoglutarate, 25 ; $o$-aminobenzaldehyde, 5; sodium carbonate buffer pH 10.25, 250; pyridoxal 5'-phosphate, 0.25 ; and crude extract equivalent to $\mathrm{I} \cdot 5 \mathrm{mg}$ protein. The reaction was started with L-lysine ( $100 \mu \mathrm{mol}$ ) or cadaverine $(300 \mu \mathrm{mol})$. After incubation at $37^{\circ} \mathrm{C}$ for $8 \mathrm{~min}$, the reaction was stopped by adding $0.5 \mathrm{ml}$ trichloroacetic acid $\left(200 \mathrm{~g} \mathrm{l}^{-1}\right)$. Precipitated protein was removed by filtration and the extinctions of the dehydroquinazolinium derivatives were measured at $465 \mathrm{~nm}$ after $90 \mathrm{~min}$ (with lysine) or $60 \mathrm{~min}$ (with cadaverine); controls lacking the oxoacid were included. Specific activities were calculated using $\epsilon_{465}=28001 \mathrm{~mol}^{-1} \mathrm{~cm}^{-1}$ for the chromogens obtained with the lysine and cadaverine products. This extinction coefficient was determined by relating the disappearance of I-piperideine to NADH formation in the I-piperideine dehydrogenase reaction. With other amino donors, the amounts added to the reaction mixtures were $50 \mu \mathrm{mol} \mathrm{L}$-ornithine and $20 \mu \mathrm{mol}$ putrescine, and the extinctions were measured after $90 \mathrm{~min}$ at $440 \mathrm{~nm}$ and $430 \mathrm{~nm}$ respectively, using $\epsilon=1860 \mathrm{l} \mathrm{mol}^{-1} \mathrm{~cm}^{-1}$ (Holmstedt, Larsson \& Tham, I96I).

I-Piperideine dehydrogenase [EC. I.5.I.-; I-piperideine:NAD ${ }^{+}$oxidoreductase] was assayed by a method analogous to that used for I-pyrroline (4-aminobutyraldehyde) dehydrogenase (Jacoby \& Fredericks, 1959). The reaction mixture contained ( $\mu \mathrm{mol}$ in $3 \mathrm{ml}$ final volume): NAD, 2; I-piperideine, 2.5; 2-mercaptoethanol, I5; Tris/ $\mathrm{HCl}$ buffer $\mathrm{pH} 9 \cdot 0$, I 50 ; and $0.35 \mathrm{mg}$ protein from the soluble fraction of crude extract. The reaction was started with I-piperideine and the increase in extinction at $340 \mathrm{~nm}$ was recorded at $25^{\circ} \mathrm{C}$ against a control without I-piperideine.

L-2-Aminoadipate aminotransferase [EC. 2.6. I . 39; L-2-aminoadipate: 2-oxoglutarate aminotransferase] and 5-aminovalerate aminotransferase [EC. 2.6.1.-; 5-aminovalerate:2oxoglutarate aminotransferase] were assayed in crude extracts at $37^{\circ} \mathrm{C}$ by a discontinuous method modified from Umbarger \& Umbarger (1962). This is based on estimating the rate 
of L-glutamate formation. The reaction mixtures contained ( $\mu \mathrm{mol}$ in $\mathrm{I} \mathrm{ml}$ final volume): amino acid, 20; 2-oxoglutarate, 20; pyridoxal 5'-phosphate, 0.08 ; and sodium potassium phosphate buffer $\mathrm{pH} \mathrm{7.5,25}$. After pre-incubating the reagents at $37^{\circ} \mathrm{C}$ for I min the reaction was started by adding the crude extract ( $1.5 \mathrm{mg}$ protein). Incubation was for $5 \mathrm{~min}$ and the reaction was stopped by heating at $100{ }^{\circ} \mathrm{C}$ for $3 \mathrm{~min}$. Precipitated protein was removed by centrifuging and the glutamate in the supernatant fluid was assayed with glutamate dehydrogenase and 3-acetylNAD according to Wyngaarden \& Ashton (1959).

Glutarate-semialdehyde dehydrogenase [EC. I.2.1.20; glutarate-semialdehyde:NADP+ oxidoreductase] was assayed in the soluble fraction of crude extracts at $25^{\circ} \mathrm{C}$ using Tris/ $\mathrm{HCl}$ buffer $\mathrm{pH} 8.5$ and NADP by the method of Chang \& Adams (1971), except that I mg bovine serum albumin was added to the reaction mixtures.

L-Pipecolate dehydrogenase [EC. I.5.99.3; L-pipecolate:(acceptor) oxidoreductase] was assayed at $25^{\circ} \mathrm{C}$ by the method of Rodwell (I97I) using 2,6-dichlorophenolindophenol as electron acceptor. This activity was normally found in the particulate fraction bu in $P$. multivorans it was present in the soluble fraction of the ultrasonic extracts.

L-Lysine 2-monooxygenase [EC. I.13.12.2; L-lysine:oxygen 2-oxidoreductase (decarboxylating)] was assayed in crude extracts at $34^{\circ} \mathrm{C}$ using a Beckman Oxygen Analyser (model 777) and the method of Takeda et al. (1969).

Isocitrate lyase [EC. 4.I.3.I; threo- $\mathrm{D}_{\mathrm{s}}$-isocitrate glyoxylate-lyase] was assayed in crude extracts at pH 6.8 according to the method of Dixon \& Kornberg (I959), except that ethylenediaminetetraacetate was substituted for cysteine as suggested by Kennedy \& Dilworth (1963).

Paper chromatography and high voltage electrophoresis. The reaction products of L-lysine, $\mathrm{L}-\left[\mathrm{U}-{ }^{14} \mathrm{C}\right]$ lysine and $\mathrm{DL}-\left[\mathrm{I}-{ }^{14} \mathrm{C}\right] l y s i n e$, and cadaverine metabolism were investigated by chromatography on Whatman no. I paper with solvents : A, t-butanol/formic acid $(90 \%)$ / $\mathrm{H}_{2} \mathrm{O}$ (70: 15: 15, by vol.); and $\mathrm{B}$, methanol/ $\mathrm{H}_{2} \mathrm{O} / \mathrm{HCl}(35 \%) /$ pyridine (I60:36:4.3:20, by vol.). High voltage electrophoresis was carried out on Whatman $3 \mathrm{MM}$ paper using a Savant flat-plate instrument. The buffers used were: $\mathrm{H}_{2} \mathrm{O} /$ pyridine/acetic acid, $\mathrm{pH} 6 \cdot 4$ (2792:200:8, by vol.) and I M-formic acid pH I.9; a potential of $100 \mathrm{~V} \mathrm{~cm}^{-1}$ was applied for 30 and 40 min respectively. Compounds were detected by spraying with ninhydrin $[0.25 \%(\mathrm{w} / \mathrm{v})$ in acetone] or $o$-aminobenzaldehyde [0.I \% $(\mathrm{w} / \mathrm{v})$ in acetone]. Radioactive compounds were detected (before spraying) with an Actigraph III radiochromatogram scanner (Nuclear Chicago) or by autoradiography. Compounds were identified by comparison with the behaviour of authentic samples under several different conditions.

Materials. DL-Pipecolic acid was prepared by catalytic hydrogenation of $\alpha$-picolinic acid hydrochloride (Rodwell, 197I) and purified as the hydrochloride by repeated recrystallization from methanol by the addition of acetone. The product was judged pure by melting point, infrared and n.m.r. spectra, and quantitative amino-acid analysis. I-Piperideine and I-pyrroline were prepared from L-lysine and L-ornithine by the method of Jacoby \& Fredericks (1959). Glutarate semialdehyde was prepared from DL-2-aminoadipic acid by the method of Chang \& Adams (1971). Glutamate dehydrogenase and 3-acetylNAD were from Boehringer, and all the other reagents and chemicals were of the highest purity obtainable commercially. 


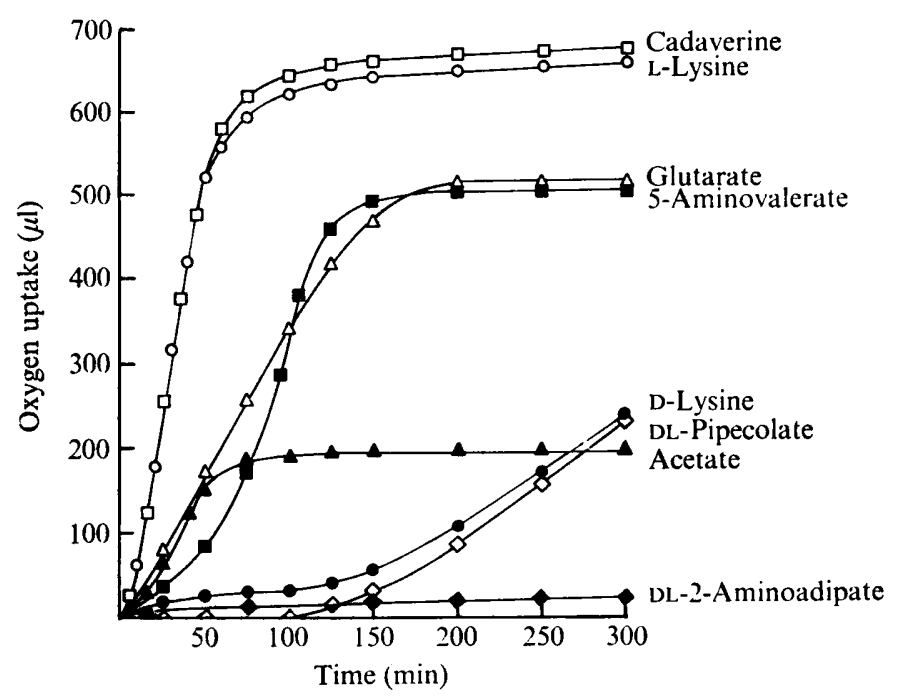

Fig. 2. Respiration of different substrates by washed suspensions of L-lysine-grown Pseudomonas aeruginosa PAC586. Exponential phase cultures were washed and bacteria equivalent to $3 \mathrm{mg}$ dry wt were incubated at $37^{\circ} \mathrm{C}$ in $0.05 \mathrm{M}$-phosphate buffer $(95 \mu \mathrm{mol}, \mathrm{pH} 7 \cdot 0)$ and substrate $(6 \mu \mathrm{mol})$ was tipped from the side-arm to give a final volume of $2.9 \mathrm{ml} . \mathrm{CO}_{2}$ was absorbed by $0.1 \mathrm{ml}$ of KOH $\left(400 \mathrm{~g} \mathrm{l}^{-1}\right)$ in the centre well. Oxygen consumption was measured manometrically in an atmosphere of air and the results were corrected for endogenous respiration, typically $1_{3} \mu \mathrm{I} \mathrm{O}_{2}$ (mg dry $w t)^{-1} h^{-1}$. The substrates were: $O$, L-lysine; $\square$, cadaverine; $\square$, 5-aminovalerate; $\triangle$, glutarate; $\boldsymbol{\Delta}$, acetate; $\boldsymbol{\ominus}$, D-lysine; $\diamond$, DL-pipecolate; $\diamond$, DL-2-aminoadipate.

\section{RESULTS}

\section{Studies with whole organisms}

In preliminary experiments 17 out of 23 different strains of Pseudomonas aeruginosa were found capable of using L-lysine as sole carbon and energy source. These included $P$. aeruginosa PAOI and $P$. aeruginosa PACI. Their specific growth rates on L-lysine minimal medium were always low $\left(\mu \leqslant 0.035 \mathrm{~h}^{-1}\right)$ compared with those observed for strains of $P$. putida, $P$. fluorescens and P. multivorans $\left(\mu=0.35\right.$ to $\left.0.09 \mathrm{~h}^{-1}\right)$. However, stable faster-growing derivatives were readily obtained either by repeated subculture in L-lysine minimal medium or by direct selection of the larger colonies which appeared when plates of solid medium were spread with $10^{9}$ organisms. Pseudomonas aeruginosa PAC586 $\left(\mu=0 \cdot 14^{\mathrm{h}^{-1}}\right)$ was obtained from the parental strain PACI $\left(\mu=0.03 \mathrm{~h}^{-1}\right)$ by the latter method and was used for studying L-lysine catabolism in P. aeruginosa. Both strains (PACI and PAC586) grew at the same specific growth rates $\left(\mathrm{h}^{-1}\right)$ on cadaverine $(0 \cdot 14), 5$-aminovalerate $(0 \cdot 3)$, glutarate $(0 \cdot 4)$, DL-pipecolate (0.9), acetate $(0.4)$, succinate $(0.4)$ and putrescine $(0.4)$, but no growth could be detected on 2-aminoadipate. Mutant PAC586 readily oxidized L-lysine, cadaverine, glutarate and acetate after growth on L-lysine (Fig. 2). 5-Aminovalerate was oxidized at an accelerating rate but it was still oxidized at a significant linear rate in the presence of chloramphenicol $\left(50 \mu \mathrm{g} \mathrm{ml}^{-1}\right)$. In contrast, 2-aminoadipate was not oxidized and D-lysine and DL-pipecolate were only oxidized after considerable lag periods (Fig. 2). The $Q_{\mathrm{O}_{2}}$ for Llysine varied from 200 to $350 \mu 1 \mathrm{O}_{2}$ (mg dry wt organisms) $)^{-1} \mathrm{~h}^{-1}$ in different experiments and the net uptake of $\mathrm{O}_{2}$ for each substrate followed to completion was between 60 and $70 \%$ of the theoretical amount calculated for total combustion to $\mathrm{CO}_{2}$. Very similar results 
were obtained with the parental strain PACI and they suggest that L-lysine is degraded via 5-aminovalerate rather than via pipecolate and 2-aminoadipate.

Using the same substrates, comparable studies with L-lysine-grown cultures of $P$. fluorescens, $P$. putida (biotypes $\mathrm{A}$ and $\mathrm{B}$ ) and $P$. multivorans gave patterns of oxidation which differed from each other and from that observed with $P$. aeruginosa. One of the most striking differences between $P$. aeruginosa and all the other species, except $P$. fluorescens, was its ability to oxidize cadaverine without lag. Furthermore, washed suspensions of cadaverinegrown PAC586 gave the same oxidation pattern as L-lysine-grown bacteria, suggesting either that L-lysine is metabolized via cadaverine or that metabolic routes for both substrates converge on the same intermediates. With 5-aminovalerate as the growth substrate, glutarate, 5-aminovalerate, L-lysine and cadaverine were all oxidized without appreciable lag and, although the oxidation rates for glutarate and 5-aminovalerate were increased up to twofold, the rates for L-lysine and cadaverine were only one-third of those observed with L-lysine or cadaverine as growth substrates. Growth on DL-pipecolate permitted immediate and rapid oxidation of D-lysine, DL-pipecolate and 5-aminovalerate; the oxidation of cadaverine and glutarate was normal after a delay of $20 \mathrm{~min}$ but L-lysine oxidation proceeded at a uniformly low rate from the time of addition. With glutarate, acetate or succinate as the growth substrate, fairly long periods (60 to $100 \mathrm{~min}$ ) of adaptation preceded the oxidation of L-lysine, DL-pipecolate, cadaverine and 5-aminovalerate by mutant PAC586.

Further studies with washed suspensions of L-lysine-grown $P$. aeruginosa PAC586 confirmed the importance of cadaverine in L-lysine catabolism. Suspensions of organisms (I mg dry wt ml-1) were incubated aerobically at $37^{\circ} \mathrm{C}$ with L-lysine $(20 \mathrm{mM})$ in phosphate buffer (Io $\mathrm{mm} ; \mathrm{pH} \mathrm{7.0)}$ and samples were withdrawn at 20 min intervals over $3 \mathrm{~h}$. Chromatographic (solvents A and B) and electrophoretic (pH 6.4) analyses of the supernatant fluids showed that as the lysine disappeared the major ninhydrin-positive material to accumulate was cadaverine, which was itself degraded on further incubation. Other minor components were detected and three of these were tentatively identified as I-piperideine, 5-aminovalerate and glutamate. In similar experiments using $\mathrm{L}-\left[\mathrm{U}-{ }^{14} \mathrm{C}\right] \mathrm{lysine},\left[{ }^{14} \mathrm{C}\right]$ cadaverine and five other labelled products were detected. With $\mathrm{DL}-\left[\mathrm{I}-{ }^{14} \mathrm{C}\right]$ lysine as substrate, no labelled cadaverine was formed (although the presence of cadaverine was confirmed with ninhydrin); three labelled products were detected but two of these were presumed to be specific metabolites of D-lysine because they differed from those formed with L-lysine.

\section{Enzymology of L-lysine catabolism in P. aeruginosa}

Extracts of L-lysine-grown $P$. aeruginosa PAC586 were examined for enzyme activities which could be responsible for the catabolism of L-lysine. No L-lysine oxygenase could be detected, nor could significant oxidase or dehydrogenase activities for L-lysine (or DLpipecolate) be found, using a variety of conditions and electron acceptors. L-Lysine aminotransferase activity was detected but L-lysine decarboxylase was the most active enzyme using L-lysine as substrate (Table I, column I). Further metabolism of the decarboxylation product, cadaverine, did not appear to involve an amine or diamine oxidase, but an aminotransferase which converted cadaverine to I-piperideine (5-aminoglutaraldehyde) was present. Further investigations revealed the presence of a NAD-dependent dehydrogenase which oxidized I-piperideine to 5-aminovalerate. This product could then be converted to glutarate in two steps by enzymes of the 5-aminovalerate (or oxygenase) pathway, 5 -aminovalerate aminotransferase and the NADP-dependent glutarate semialdehyde dehydrogenase, which were present at high specific activity (Table I, column I). Glutaryl-CoA synthase activity could not be detected but it was assumed from the relatively high specific 
Table I. Enzyme activities detected in ultrasonic extracts of Pseudomonas aeruginosa PAC586 after growth on L-lysine and other substrates

Cultures were grown with different substrates as sole carbon and energy sources and enzymes assayed as described in Methods. Specific activities are recorded as $\mu$ mol substrate transformed (mg protein) $)^{-1} \mathrm{~h}^{-1}$.

Specific activity after growth on:

\section{Enzyme}

L-Lysine decarboxylase

Cadaverine aminotransferase

I-Piperideine dehydrogenase

5-Aminovalerate aminotransferase

Glutarate-semialdehyde dehydrogenase

Isocitrate lyase

L-Lysine 6-aminotransferase

L-2-Aminoadipate aminotransferase

\begin{tabular}{|c|c|c|c|c|c|c|c|c|c|}
\hline 壳 & 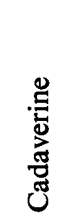 & 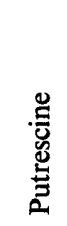 & 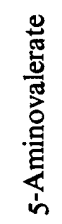 & 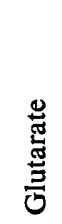 & 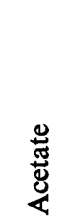 & 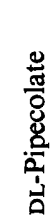 & 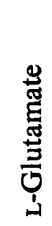 & 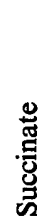 & 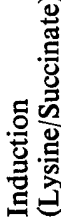 \\
\hline $7 \cdot 5$ & $9 \cdot I$ & $0 \cdot I$ & $3 \cdot 3$ & 0.5 & 0.3 & 7.5 & 0.2 & $0 \cdot I$ & 75 \\
\hline 3.5 & $3 \cdot 2$ & $5 \cdot 2$ & $3 \cdot 2$ & $2 \cdot I$ & 0.6 & $I \cdot O$ & $1 \cdot 0$ & 0.3 & 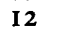 \\
\hline 0.0 & $9 \cdot I$ & 14.6 & 6.9 & $5 \cdot I$ & $\mathbf{I} \cdot \mathbf{I}$ & 3.6 & $I \cdot 2$ & $I \cdot 3$ & \\
\hline $7 \cdot 5$ & $7 \cdot 7$ & $6 \cdot 8$ & $7 \cdot 9$ & $6 \cdot I$ & $\mathbf{I} \cdot \mathbf{I}$ & $2 \cdot 0$ & 0.5 & 0.9 & \\
\hline 7 & $32 \cdot 7$ & 25.4 & $23 \cdot 2$ & $15 \cdot I$ & $1 \cdot 2$ & $9 \cdot 2$ & 2.8 & $1 \cdot 5$ & I 8 \\
\hline I. 8 & $8 \cdot 2$ & 0.9 & $24 \cdot 2$ & 19.0 & 18.6 & 3.0 & $I \cdot 7$ & $1 \cdot 7$ & 7 \\
\hline $2 \cdot 3$ & $2 \cdot I$ & $3 \cdot 6$ & 2.5 & $\mathrm{I} \cdot 4$ & 0.3 & 0.7 & 0.4 & 0.3 & $\mathbf{0}$ \\
\hline $1 \cdot 0$ & $I \cdot 2$ & $I \cdot 3$ & $1 \cdot 3$ & 0.8 & 0.3 & 0.6 & 0.4 & 0.4 & \\
\hline
\end{tabular}

activity of isocitrate lyase that L-lysine was metabolized largely via acetyl-CoA. L-Pipecolate dehydrogenase, a key enzyme of the pipecolate pathway, was just detectable and L-2-aminoadipate aminotransferase was present but not at a high specific activity.

These results indicated that L-lysine may be degraded by a new pathway, the cadaverine pathway, which is initiated by decarboxylation of the amino acid and converges with the oxygenase pathway at 5 -aminovalerate after the diamine has been transaminated to Ipiperideine (Fig. 3). It can be calculated that the specific activities are sufficient to support the growth of mutant PAC586 on L-lysine at the observed rate. Another route initiated by transamination may also be present. New routes involving racemization to D-lysine were not excluded.

Some of the relevant enzyme activities were studied in more detail.

L-Lysine decarboxylase. Lysine decarboxylation by crude extracts was entirely dependent on the presence of pyridoxal 5'-phosphate, and the addition of 2-mercaptoethanol further improved the rate of decarboxylation by a factor of $\mathrm{I} \cdot 7$. The optimum $\mathrm{pH}$ was 6.8 and activity declined to very low values at $\mathrm{pH} 6.0$ and $\mathrm{pH} 9 \cdot 0$. The $K_{\mathrm{m}}$ for L-lysine was Io mM and $V_{\max }$ was $9.8 \mu \mathrm{mol}(\mathrm{mg} \text { protein })^{-1} \mathrm{~h}^{-1}$. The product of the reaction was identified as cadaverine by chromatographic and electrophoretic techniques and by extraction and identification of its 2,4-dinitrophenyl derivative from reaction mixtures.

$\mathrm{L}-$ Lysine and cadaverine aminotransferases. These aminotransferases were assayed in extracts of L-lysine-grown organisms with 2-oxoglutarate as the amino acceptor and $o$ aminobenzaldehyde present. The optimum $\mathrm{pH}$ was 9.8 with both substrates. Both activities declined steadily at higher $\mathrm{pH}$ values, the rates being half-maximum at $\mathrm{pH} 10.5$, but at lower $\mathrm{pH}$ values the rates with cadaverine fell more steeply (half-maximum at $\mathrm{pH} \mathrm{9.3)} \mathrm{than} \mathrm{the}$ rates with lysine (half-maximum at $\mathrm{pH} 8.8$ ). Very little lysine decarboxylase activity was detected above $\mathrm{pH} 9.0$ so the aminotransferase activity observed with lysine is specific for lysine and not due to the combined activities of lysine decarboxylase and cadaverine aminotransferase. No activity could be detected when 2-oxoglutarate was omitted, except in the 


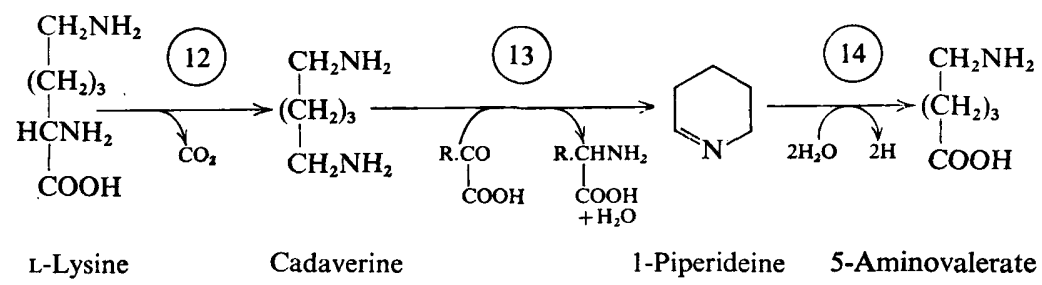

Fig. 3. Scheme showing the proposed cadaverine (decarboxylase) pathway of L-lysine catabolism. The enzymes involved are: (12) L-lysine decarboxylase; (13) cadaverine aminotransferase; (14) I-piperideine dehydrogenase. I-Piperideine is in equilibrium with the hydrated open structure, 5-aminoglutaraldehyde.

$\mathrm{pH}$ range 7 to 9 where very weak lysine and cadaverine oxidative deaminations seemed to occur. The activities of crude extracts were increased by approximately 10 \% after passing through small columns of either Sephadex G-25 or Dowex-I (X8, I00 to $200 \mathrm{mesh}$; $\mathrm{H}^{+}$ form). No requirement for pyridoxal 5 -phosphate could be detected but it was routinely added to the reaction mixtures.

2-Oxoglutarate was the most active amino acceptor tested $\left(K_{\mathrm{m}}=\mathrm{I} \mathrm{mm}\right)$. Glyoxylate, pyruvate and oxaloacetate gave between 10 and $40 \%$ of the activities obtained with 2-oxoglutarate under comparable conditions. Studies with a range of amino donors showed that, in addition to L-lysine $\left(K_{\mathrm{m}}=\right.$ I I $\left.\mathrm{mM}\right)$ and cadaverine $\left(K_{\mathrm{m}}=67 \mathrm{mM}\right)$, much higher affinities for transamination by the crude extracts were found with putrescine $\left(K_{\mathrm{m}}=0.8 \mathrm{~mm}\right)$ and ornithine $\left(K_{\mathrm{m}}=1.7 \mathrm{mM}\right)$. However, the values for $V_{\max }$ were very similar with all four substrates, ranging from 2 to $6 \mu \mathrm{mol}$ (mg protein) ${ }^{-1} \mathrm{~h}^{-1}$ with $6 \mathrm{~mm}$-2-oxoglutarate.

Transamination of lysine could involve the 2- or 6-amino groups yielding I-piperideine-2carboxylate or I-piperideine-6-carboxylate respectively. These differ in several respects (Soda et al., 1968). The 2-carboxylate reacts rapidly with $o$-aminobenzaldehyde to produce a chromogen with an extinction maximum at $450 \mathrm{~nm}$. The 6-carboxylate reacts slowly with $o$-aminobenzaldehyde and the corresponding chromogen has an extinction maximum at $465 \mathrm{~nm}$; it also reacts rapidly with ninhydrin in glacial acetic acid to give a stable yellow complex. The two compounds also exhibit different electrophoretic mobilities in I Mformic acid. Based on these criteria, the reaction product was shown to be I-piperideine-6carboxylate, i.e. the enzyme activity corresponds to L-lysine 6-aminotransferase. Deproteinized samples of reaction mixtures containing L- $\left[\mathrm{U}-{ }^{14} \mathrm{C}\right]$ lysine or DL- $\left[\mathrm{I}-{ }^{14} \mathrm{C}\right]$ lysine plus $2-$ oxoglutarate incubated with crude extract at $\mathrm{pH} 9.8$ were also examined and electrophoretic analysis confirmed that the major radioactive product corresponded to I-piperideine-6carboxylate in both cases. The product of cadaverine transamination was judged to be Ipiperideine by comparing the properties of its $o$-aminobenzaldehyde adduct with that of synthetic I-piperideine. The formation of glutamate from 2-oxoglutarate with lysine and cadaverine was demonstrated by quantitative studies using glutamate dehydrogenase. Furthermore, this procedure provided an alternative but less convenient method for assaying the aminotransferases. It is not certain whether the lysine and cadaverine aminotransferase activities represent two discrete enzymes or one or more enzymes with wide substrate specificities. However, in this context it should be noted that the L-lysine 6-aminotransferase of Achromobacter liquidum was inactive with cadaverine (Soda \& Misono, 1968) and the diamine aminotransferase of Escherichia coli was likewise inactive with lysine.

I-Piperideine dehydrogenase. This enzyme was best assayed by following the rate of NADH formation using the soluble fraction to limit NADH oxidase activity. The $K_{\mathrm{m}}$ for I-piperi- 
deine was $0.12 \mathrm{mM}$ and $V_{\max }$ was $12 \mu \mathrm{mol}$ (mg protein) $)^{-1} \mathrm{~h}^{-1}$. The enzyme could also be assayed discontinuously by measuring the disappearance of I-piperideine using $o$-aminobenzaldehyde. A direct relationship between I-piperideine disappearance and NADH formation was established and this was used to determine the molar extinction coefficient for the dehydroquinazolinium derivative. The coenzyme NADP could replace NAD but it gave activities which were only $10 \%$ of those obtained with NAD under comparable conditions. This contrasts with glutarate semialdehyde where NADP produced to times more activity than NAD. Attempts to demonstrate a 5-aminovalerate-dependent oxidation of NADH by the reverse reaction were unsuccessful. I-Pyrroline was also oxidized by these extracts and, using the same conditions, the rate with this substrate (optimum at $\mathrm{pH} 7.5$ ) was about one-third of the rate observed with I-piperideine (optimum at $\mathrm{pH} 9 \cdot 0$ ). Extracts of putrescine-grown organisms possessed high I-piperideine dehydrogenase (Table I) and $\mathrm{I}$-pyrroline dehydrogenase activities in the same proportion as found in lysine-grown organisms. However, it is not known whether one or two dehydrogenases are involved.

\section{Status of L-lysine-degrading enzymes in P. aeruginosa PAC586 grown on different substrates}

In order to assess the importance of the proposed cadaverine pathway for L-lysine catabolism in $P$. aeruginosa PAC586, the specific activities of relevant enzymes were determined in organisms grown on a range of substrates (Table $I$ ). The degree of induction for each enzyme is shown as the increase in activity in L-lysine-grown cultures relative to control cultures grown with succinate. All the relevant enzymes were induced by factors of 7 to 75 and this is consistent with the existence of an inducible pathway for L-lysine degradation via cadaverine, 5-aminovalerate, glutarate and acetyl-CoA. A very similar pattern of induction was observed with cultures grown on cadaverine and 5-aminovalerate, although in the latter case the induction of lysine decarboxylase was less pronounced. Growth on putrescine induced all the activities except the decarboxylase and isocitrate lyase, and with arginine (not shown) only isocitrate lyase was not induced. Cultures grown on glutarate contained even less lysine decarboxylase but still retained reasonably high levels of the other enzymic activities. In contrast, the acetate-grown organisms were only induced for isocitrate lyase. The results obtained are in reasonable agreement with the respiratory capacities observed with whole organisms grown on the same substrates. One exception appeared to be the DL-pipecolate-grown cultures which were highly induced for lysine decarboxylase but oxidized L-lysine very slowly. On the other hand, the cadaverine and 5-aminovalerate aminotransferases were low considering that these substrates were oxidized fairly readily. Attempts to compare and interpret such data are frustrated by the problems of cell permeability and also the unknown substrate specificities of enzymes assayed in crude extracts. Thus, it is possible that the observed I-piperideine dehydrogenase activity of DL-pipecolate-grown cultures is really due to a soluble pipecolate dehydrogenase. The simplest interpretation of the high glutarate-semialdehyde dehydrogenase is that pipecolate is metabolized via glutarate semialdehyde or glutarate, but it could be due to a wide substrate specificity of a 2aminoadipate semialdehyde dehydrogenase. The weak induction of isocitrate lyase compared with glutarate-grown cultures may suggest that pipecolate is not primarily metabolized via glutarate and acetyl-CoA. L-2-Aminoadipate aminotransferase was only weakly induced by growth on L-lysine and pipecolate. The induction of L-lysine 6-aminotransferase parallels the induction of other enzymes of the cadaverine pathway, particularly cadaverine aminotransferase (Table I), but it is not possible to interpret this in terms of one or two discrete 
aminotransferases. However, discrete enzymes may exist because lysine is not a substrate for the diamine aminotransferase of $E$. coli and cadaverine is not transaminated by the lysine aminotransferase of Achromobacter liquidum.

\section{Studies with mutants of $P$. aeruginosa PAC586}

Mutants of $P$. aeruginosa PAC586 which grew with succinate but not L-lysine as sole carbon and energy sources were isolated after mutagenesis and enrichment by penicillin selection (see Methods). Growth tests with 23 mutants isolated in one experiment indicated the presence of three basic groups. The largest group (Lut ${ }^{-}$), comprising 13 mutants, closely resembled the parental strain PACI in growing well on cadaverine, 5-aminovalerate, glutarate and acetate, but growing very slowly on L-lysine. Another group (Gut $\left.{ }^{-}\right)$, with eight representatives, grew on acetate but failed to grow on glutarate, cadaverine, pipecolate, 5aminovalerate or lysine. Further tests showed that six of the Gut- mutants failed to grow on glutaconate but grew on butyrate, whereas the remaining two mutants did not grow with either substrate. Consequently those growing on butyrate could have lesions affecting the conversion of glutaconyl-CoA to crotonyl-CoA whereas the other two may be blocked between crotonyl-CoA and acetyl-CoA. The third group (Aut ${ }^{-}$) consisted of two mutants which failed to grow on acetate and all the other test substrates, including pipecolate. The nutritional phenotype of the third group was also exhibited by a well-characterized isocitrate lyase mutant of $P$. aeruginosa, PAC50I. However, the two Aut $^{-}$strains contained at least $50 \%$ of the uninduced activity of isocitrate lyase of the parental strain so they were assumed to have some other lesion(s) affecting acetate metabolism.

The existence of alternative routes for L-lysine degradation to glutarate (or glutaconate) in $P$. aeruginosa PAC586 is strongly favoured by these studies. If there were just one route, mutants with the phenotypes expected for metabolic blocks between intermediate substrates cadaverine, 5-aminovalerate and glutarate should have been recovered. It may be possible to obtain such mutants by specific selections for failure to grow on cadaverine or 5-aminovalerate, and the existence of alternative routes of lysine catabolism would be confirmed if these mutants were capable of growing on L-lysine. The results clearly show that a functional glutarate catabolic pathway is essential for the catabolism of L-lysine, cadaverine and 5aminovalerate and that glutarate in turn is degraded via acetyl-CoA. Also, despite the low activities of isocitrate lyase in DL-pipecolate-grown cultures, this enzyme and others involved in glutarate catabolism are essential for pipecolate metabolism, which appears to converge on glutarate (or glutaconate) and hence acetyl-CoA in $P$. aeruginosa.

\section{L-Lysine catabolism in other species}

The occurrence and significance of the cadaverine pathway in other species of Pseudomonas was investigated with L-lysine-grown bacteria and their extracts. Studies on the oxidation of relevant substrates (see Fig. 2) by washed suspensions gave some indication of the routes of lysine catabolism which might be induced in different species. For example, $P$. fluorescens oxidized all substrates including cadaverine, D- and L-lysine, DL-pipecolate and 5-aminovalerate without lag, indicating that several pathways may be operating. With $P$. multivorans only D- and L-lysine, DL-pipecolate and L-2-aminoadipate were oxidized rapidly, suggesting that the major route for lysine catabolism is the pipecolate pathway; oxidation of cadaverine, glutarate, 5 -aminovalerate and acetate was very slow and was always preceded by long periods of adaptation. It was difficult to interpret the results for the representatives of $\boldsymbol{P}$. putida biotypes A and B. Neither oxidized cadaverine without lag. However, both were induced for D-lysine and acetate oxidation but DL-pipecolate was oxi- 
Table 2. Enzymes of L-lysine catabolism in different species of Pseudomonas

Organisms were grown on L-lysine at $37^{\circ} \mathrm{C}\left(P\right.$. aeruginosa) or $30^{\circ} \mathrm{C}$ (all the other species), harvested in the exponential phase and extracts were assayed for enzyme activities as described in Methods. The results shown in parentheses were obtained under anaerobic conditions for L-lysine decarboxylase or with the soluble fraction for L-pipecolate dehydrogenase.

Specific activity of enzyme in extracts of:

\begin{tabular}{|c|c|c|c|c|c|c|}
\hline Enzyme & $\begin{array}{l}\text { aerugino } \\
\text { PAC586 }\end{array}$ & $\begin{array}{l}\text { aeruginosa } \\
\text { PACI }\end{array}$ & $\begin{array}{l}\text { P. fluores- } \\
\text { cens }\end{array}$ & $\begin{array}{c}P . \text { putida } \\
\text { (biotype A) }\end{array}$ & $\begin{array}{c}P . \text { putida } \\
\text { (biotype в) }\end{array}$ & $\begin{array}{l}\text { P. multi- } \\
\text { vorans }\end{array}$ \\
\hline L-Lysine decarboxylase & $7 \cdot 5$ & $2 \cdot 3$ & $\begin{array}{c}7 \cdot 0 \\
(5 \cdot 5)\end{array}$ & $\begin{array}{l}0.9 \\
(0.03)\end{array}$ & $\begin{array}{l}I \cdot 0 \\
(0.06)\end{array}$ & I.5 \\
\hline Cadaverine aminotransferase & $3 \cdot 5$ & $2 \cdot 6$ & $2 \cdot 6$ & $I \cdot I$ & $2 \cdot 6$ & 0.5 \\
\hline I-Piperideine dehydrogenase & $10 \cdot 0$ & $7 \cdot 4$ & $9 \cdot 2$ & $1 \cdot 0$ & $6 \cdot 8$ & 0.3 \\
\hline $\begin{array}{l}\text { 5-Aminovalerate } \\
\text { aminotransferase }\end{array}$ & $7 \cdot 5$ & $4 \cdot 3$ & $3 \cdot 7$ & $1 \cdot 4$ & $4 \cdot 3$ & $<0.2$ \\
\hline $\begin{array}{l}\text { Glutarate-semialdehyde } \\
\text { dehydrogenase }\end{array}$ & $27 \cdot 2$ & 19.9 & $15 \cdot 9$ & $5 \cdot 5$ & $27 \cdot 9$ & $2 \cdot 6$ \\
\hline Isocitrate lyase & II $\cdot 8$ & 14.4 & $15 \cdot 2$ & $3 \cdot 9$ & $12 \cdot 7$ & $2 \cdot 3$ \\
\hline L-Lysine 6-aminotransferase & $2 \cdot 3$ & $1 \cdot 5$ & $1 \cdot 5$ & 0.5 & $2 \cdot I$ & 0.5 \\
\hline L-Lysine 2-monooxygenase & $<0.05$ & $<0.05$ & $12 \cdot 6$ & 44 & $4 \cdot 2$ & $<0.05$ \\
\hline L-Pipecolate dehydrogenase & 0.02 & $<0.01$ & 0.02 & 0.9 & 0.03 & $\begin{array}{l}0.02 \\
(0.15)\end{array}$ \\
\hline L-2-Aminoadipate & $1 \cdot 0$ & $1 \cdot 6$ & 0.8 & $1 \cdot 5$ & $0 \cdot 8$ & $6 \cdot 8$ \\
\hline
\end{tabular}

dized only by $P$. putida A although $P$. putida B was better adapted for oxidizing 5-aminovalerate.

Using extracts of L-lysine-grown organisms, enzymes of the cadaverine pathway and other relevant enzymes were assayed in several Pseudomonas species and in $P$. aeruginosa PACI, the parent (Lut-) of strain PAC586 $\left(\right.$ Lut $\left.^{+}\right)$(Table 2). The parental strain had a similar distribution of enzyme activities to that of its derivative PAC586. Some of the enzymes, particularly L-lysine decarboxylase, were present at lower specific activities in strain PACI.

In assessing the significance of the cadaverine pathway in organisms containing L-lysine 2-monooxygenase it was important to assay the decarboxylase under anaerobic conditions because the oxygenase also liberates $\mathrm{CO}_{2}$. In $P$. aeruginosa the specific activities for L-lysine decarboxylase were the same under aerobic and anaerobic conditions. However, in $P$. fluorescens $20 \%$ of the apparent decarboxylase activity could be attributed to the oxygenase and in $P$. putida of biotypes A and B the oxygenase could account for virtually all the decarboxylation (Table 2). The results indicate that $P$. fluorescens is potentially capable of metabolizing L-lysine by decarboxylation to cadaverine as well as by oxygenation. In contrast, the cadaverine pathway did not appear to be operating in either strain of $P$. putida. The oxygenase pathway was present and, in the representative of the A biotype, pipecolate dehydrogenase was also induced. In $P$. multivorans no oxygenase could be detected and, although a small amount of L-lysine decarboxylase activity was observed, other enzymes of the cadaverine and 5-aminovalerate routes were present at very low specific activities. A 'soluble' pipecolate dehydrogenase was detected and the high specific activity for L-2aminoadipate aminotransferase confirmed the indications from studies with whole cells, that the pipecolate route is probably important for L-lysine catabolism in P. multivorans. All the species tested possessed L-lysine aminotransferase activity. 


\section{DISCUSSION}

The report of Stanier et al. (1966) that $90 \%$ (26/29) of strains of Pseudomonas aeruginosa use L-lysine as sole carbon and energy source was confirmed by finding that $74 \%(\mathrm{I} 7 / 23)$ of the strains studied here possess this phenotype. These growth tests conceal the fact that the rate of growth on L-lysine is normally low. However, faster growing derivatives were readily isolated. Studies with extracts of one derivative, $P$. aeruginosa PAC586, indicated that it was deficient in key enzymes of the pathways used for D- and L-lysine catabolism in other Pseudomonas species. A search for alternative routes led to the discovery of reactions which constitute a new pathway, the cadaverine or decarboxylase pathway. This represents a variant on the 5-aminovalerate (or oxygenase) pathway because it converges on 5-aminovalerate but involves both acyclic (cadaverine) and cyclic (I-piperideine) intermediates which by-pass the route involving 5 -aminovaleramide and the monooxygenase and amidase reactions (Figs I and 3). Enzymes for the conversion of 5-aminovalerate to glutarate were present, as in other species of Pseudomonas grown on L-lysine.

The cadaverine pathway (Fig. 3) is initiated by L-lysine decarboxylase. This enzyme was not detected in $P$. aeruginosa in early studies (Gale, 1946). One reason may have been the use of a low $\mathrm{pH}$ for the tests and this could explain the continued appearance of conflicting reports. For example, no lysine decarboxylase was detected in the $\boldsymbol{P}$. aeruginosa strains tested by Steel \& Midgley (I962) and Zolg \& Ottow (1974) although all of I 30 different cultures of $\boldsymbol{P}$. aeruginosa were found to possess this activity in tests at $\mathrm{pH} \mathrm{8.0} \mathrm{(Stewart,}$ 1970). The ability of $P$. aeruginosa to oxidize cadaverine and other diamines by inducible enzymes is well established (Gale, 1942; Razin, Gery \& Bachrach, 1959). The route proposed for the further metabolism of cadaverine to glutarate is directly analogous to that for the metabolism of putrescine to succinate via I-pyrroline, 4-aminobutyrate and succinate semialdehyde in $P$. fluorescens and $E$. coli (Jacoby \& Fredericks, 1959; Kim, 1964). The analogy may be extended because putrescine is the decarboxylation product of ornithine and consequently occurs as an intermediate in the catabolism of arginine and ornithine as well as spermine and spermidine in some pseudomonads. Growth of $P$. aeruginosa PAC586 on putrescine and arginine induced the enzymes for the conversion of cadaverine to glutarate, suggesting that either one group of enzymes, or two groups with overlapping specificities, may be responsible for the catabolism of the diamine. It is difficult to assess the most likely possibility because some of the relevant enzymes have fairly broad substrate specificities. Arginine, but not putrescine, also induced some lysine decarboxylase activity. The importance of cadaverine as an intermediate with regulatory significance is apparent from the fact that the respiratory activities (Fig. 2) and enzyme patterns (Table I) of cadaverineand lysine-grown cultures of mutant PAC586 are identical. Despite the difference in growth rates on L-lysine of the mutant PAC586 and its parent strain, PACI, both grew at the same rate on cadaverine. The specific activity of lysine decarboxylase in lysine-grown strain PACI was less than one-third of that of mutant PAC586 and the slower growth of strain PACr may be a direct consequence of this. Alternatively, the growth of strain PACI may be retarded by a deficiency in the L-lysine transport system which limits the internal concentration of Llysine and hence the degree of induction of the catabolic enzymes. However, it should be emphasized that synthesis of the decarboxylase is still inducible in the mutant PAC586. Pseudomonas aeruginosa may therefore be seen as having a very efficient inducible system(s) for diamine metabolism, and rapid growth on L-lysine can be accomplished by improving the flow of this substrate into the diamine pathway. In this respect L-lysine catabolism resembles L-histidine catabolism in $P$. aeruginosa strain PACI, where histidine transport is 
sufficient for its utilization as a source of nitrogen but not for supporting growth as sole carbon source (Potts \& Clarke, 1974).

The transamination of L-lysine to I-piperideine-6-carboxylate is also inducible and represents a second potential route for lysine catabolism in $P$. aeruginosa. Its physiological significance is difficult to assess. It could represent a mechanism for obtaining aminonitrogen when $\boldsymbol{P}$. aeruginosa is grown with other substrates plus L-lysine as sole source of nitrogen. The failure to isolate mutants unable to utilize lysine due to blocks between Llysine and glutarate implies that there are at least two alternative and functional routes effecting the corresponding metabolic transformation. As an intermediate in the pipecolate pathway (Fig. I), it might be assumed that I-piperideine-6-carboxylate would be metabolized via 2-oxoglutarate. However, the dependence on a functional glutarate pathway for growth on L-lysine and DL-pipecolate and the induction of isocitrate lyase with both substrates imply that the product of the pipecolate pathway, 2-oxoadipate, can be converted to glutarate (or glutaryl-CoA) or that one of the $\mathrm{C}_{6}$ compounds (I-piperideine-6-carboxylate or 2-aminoadipate) can be decarboxylated to converge on the 5 -aminovalerate route (at piperideine or 5-aminovalerate). Studies with mutants specifically selected for blocks in cadaverine or 5-aminovalerate metabolism could be used for assessing the significance of the transamination route and the location of any metabolic bridge between the $\mathrm{C}_{6}$ and $\mathrm{C}_{5}$ routes. The affinities of some of the enzymes for their substrates appear low and their $\mathrm{pH}$ optima are rather high. However, high $\mathrm{pH}$ optima are often observed for enzymes concerned with the metabolism of basic substrates and high Michaelis constants for substrates of inducible pathways concerned with the catabolism of essential metabolites such as lysine are obviously advantageous (Miller \& Rodwell, 1971 $a, b$ ).

The results obtained with $P$. fluorescens indicate that it has the potential for using the cadaverine pathway; its existence may have been overlooked in previous studies due to the presence of the high lysine oxygenase activity. Pseudomonas multivorans had a weak decarboxylase activity and this has been noted previously in three strains including the one studied here (Samuels, Moss \& Weaver, 1973). However, neither the cadaverine nor other routes involving 5-aminovalerate appear important, and this strain could rely entirely on the pipecolate route for lysine catabolism. Decarboxylation does not appear to be important in the catabolism of L-lysine by $P$. putida. The results are consistent with those of Chang \& Adams (1974) and Miller \& Rodwell (I97I $a$ ); the oxygenase and pipecolate dehydrogenase routes both occur in strains of biotype $A$. The oxygenase route seems to be the main route present in the representative of biotype $\mathrm{B}$. The results also show that the role of L-lysine 6-aminotransferase needs to be considered when assessing the physiological importance of different routes for lysine catabolism in all the strains. Kornberg (1960) used the specific activity of isocitrate lyase as an enzymic indicator of the metabolic pathways involved in L-lysine (and glutarate) catabolism by another $P$. putida of biotype A. He concluded that L-lysine was degraded via acetate, not 2-oxoglutarate. Using the same criterion this is true for all the Pseudomonas species examined here, except $P$. multivorans.

In view of the relatively small number of strains which have been examined it cannot be concluded that there is a rigid specificity for the routes of lysine catabolism possessed by different Pseudomonas species. However, it is clear that not only do the pseudomonads possess great metabolic potential but there is also a great diversity in the number of pathways for the catabolism of one substrate. Furthermore, in the case of lysine catabolism, multiple pathways can exist in one strain, posing interesting regulatory problems as well as those posed by the inter-relationships between the biosynthesis and degradation of the amino acid. 
One of us (J.C.F.) acknowledges the support from an S.R.C. Research Studentship during the course of this work.

\section{REFERENCES}

Baginsky, M. L. \& Rodwell, V. W. (1966). Metabolism of pipecolic acid in a Pseudomonas species. IV. Electron transport particle of Pseudomonas putida. Journal of Bacteriology 92, 424-432.

Calvert, A. F. \& Rodwell, V. W. (I966). Metabolism of pipecolic acid in a Pseudomonas species. III. $L-\alpha$-Amino adipate - $\delta$-semialdehyde:NAD oxidoreductase. Journal of Biological Chemistry 24I, 409-414.

Chang, Y-F. \& Adams, E. (197I). Glutaric semialdehyde dehydrogenase (Pseudomonas putida). Preparation and assay of glutaric semialdehyde. Methods in Enzymology XVIIB, 166-I 73.

Chang, Y-F. \& ADAms, E. (1974). D-Lysine catabolic pathway in Pseudomonas putida: interrelations with L-lysine catabolism. Journal of Bacteriology II7, 753-764.

Dixon, G. H. \& KoRnBerg, H. L. (I959). Assay methods for key enzymes of the glyoxylate cycle. Biochemical Journal 72, 3P.

GALE, E. F. (1942). The oxidation of amines by bacteria. Biochemical Journal 36, 64-75.

GALE, E. F. (1946). The bacterial amino acid dehydrogenases. Advances in Enzymology 6, I-32.

Hartline, R. A. \& Rodwell, V. W. (1971). Metabolism of pipecolic acid in a Pseudomonas species. VI. Precursors of glutamic acid. Archives of Biochemistry and Biophysics 142, 32-39.

Holmstedt, B., Larsson, L. \& Tham, B. (1961). Further studies of a spectrophotometric method for the determination of diamine oxidase activity. Biochimica et biophysica acta 48, 182-186.

ICHIHARA, A. \& ICHIHARA, E. A. (1961). Metabolism of L-lysine by bacterial enzymes. V. Glutaric semialdehyde dehydrogenase. Journal of Biochemistry 49, $154-157$.

IChiHARA, A., FuriYa, S. \& SudA, M. (1960).' Metabolism of L-lysine by bacterial enzymes. III. Lysine racemase. Journal of Biochemistry 48, 277-282.

IChimara, A., IChirara, E. A. \& SuDA, M. (I960). Metabolism of L-lysine by bacterial enzymes. IV. $\delta$ Aminovaleric acid-glutamic acid transaminase. Journal of Biochemistry 48, 4I 2-420.

JACOBY, W. B. \& Fredericks, J. (1959). Pyrrolidine and putrescine metabolism: $\gamma$-aminobutyraldehyde dehydrogenase. Journal of Biological Chemistry 234, 2 I 45-21 50.

KenNedY, I. R. \& Dilworth, M. J. (1963). Activation of isocitrate lyase and triosephosphate dehydrogenase in Azotobacter vinelandii extracts. Biochimica et biophysica acta 67, 226-239.

KIM, K-H. (1964). Purification and properties of a diamine $\alpha$-ketoglutarate transaminase from Escherichia coli. Journal of Biological Chemistry 239, 783-786.

KORNBERG, H. L. (1960). Isocitratase as an enzymic indicator of metabolic pathways. Nature, London 188 , 488-489.

LineweAVER, H. \& BURK, D. (1934). The determination of enzyme dissociation constants. Journal of the American Chemical Society 56, 658-666.

Lowry, O. H., Rosebrough, N. J., FARR, A. L. \& Randall, R. J. (I95I). Protein measurement with the Folin phenol reagent. Journal of Biological Chemistry 193, 265-275.

Miller, D. L. \& Rodwell, V. W. (I97I $a$ ). Metabolism of basic amino acids in Pseudomonas putida. Catabolism of lysine by cyclic and acyclic intermediates. Journal of Biological Chemistry 246, 2758-2764.

Miller, D. L. \& RodWell, V. W. (1971 $b$ ). Metabolism of basic amino acids in Pseudomonas putida: intermediates in L-arginine catabolism. Journal of Biological Chemistry 246, 5053-5058.

Numa, S., Ishimura, Y., Nakazawa, D., OKazaki, T. \& Hayaishi, O. (I964). Enzymic studies on the metabolism of glutarate in Pseudomonas. Journal of Biological Chemistry 239, 391 5-3926.

PotTs, J. R. \& ClARKe, P. H. (1974). The regulation of histidine catabolism in Pseudomonas aeruginosa. Proceedings of the Society for General Microbiology $\mathbf{1}, 63$.

RAZIN, S., GERY, I. \& BACHRACH, U. (1959). The degradation of natural polyamines and diamines by bacteria. Biochemical Journal 7r, $55 \mathrm{I}-558$.

ReITZ, M. S. \& RoDWELL, V. W. (1969). $\alpha$-Hydroxyglutarate oxidoreductase of Pseudomonas putida. Journal of Bacteriology roo, 708-714.

ReItz, M. S. \& Rodwell, V. W. (1970). $\delta$-Aminovaleramidase of Pseudomonas putida. Journal of Biological Chemistry 245, 309I-3096.

Rodwell, V. W. (1971). Pipecolic acid. Methods in Enzymology XVIB, 174-I 88.

Samuels, S. B., Moss, C. W. \& WeAVER, R. E. (1973). The fatty acids of Pseudomonas multivorans (Pseudomonas cepacia) and Pseudomonas kingii. Journal of General Microbiology 74, 275-279.

Skinner, A. J. \& Clarke, P. H. (1968). Acetate and acetamide mutants of Pseudomonas aeruginosa 8602. Journal of General Microbiology 50, I83-194.

SodA, K. \& Misono, H. (1968). L-Lysine- $\alpha$-ketoglutarate aminotransferase. II. Purification, crystallization and properties. Biochemistry 7, 41 10-4I 19.

Soda, K., Misono, H. \& Yamamoto, T. (I968). L-Lysine- $\alpha$-ketoglutarate aminotransferase. I. Identification of a product, $\Delta^{\prime}$-piperideine-6-carboxylic acid. Biochemistry 7, 4102-4109. 
Stanier, R. Y., Palleroni, N. J. \& Doudoroff, M. (I966). The aerobic pseudomonads: a taxonomic study. Journal of General Microbiology 43, 159-271.

SteEL, K. J. \& MiDGLeY, J. (1962). Decarboxylase and other reactions of some Gram-negative rods. Journal of General Microbiology 29, 171-178.

STEWART, D. J. (1970). The decarboxylation of L-lysine by Pseudomonas aeruginosa isolates. Journal of Applied Bacteriology 33, 501-504.

Takeda, H., Yamamoto, S., Kojima, Y. \& Hayaishi, O. (1969). Studies on monooxygenases. I. General properties of crystalline L-lysine monooxygenase. Journal of Biological Chemistry 244, 2935-2941.

UMBARGER, H. E. \& UMBARGER, M. E. (1962). The biosynthetic pathway of serine in Salmonella typhimurium. Biochimica et biophysica acta 62, 193-195.

VANDECASTEELE, J-P. \& HeRmanN, M. (1972). Regulation of a catabolic pathway: lysine degradation in Pseudomonas putida. European Journal of Biochemistry 31, 80-85.

WyngaARDEN, J. B. \& Ashton, D. M. (I959). The regulation of activity of phosphoribosylpyrophosphate amidotransferase by purine ribonucleotides: a potential feedback control of purine biosynthesis. Journal of Biological Chemistry 234, 1492-1496.

ZolG, W. \& OTtow, J. C. G. (1974). Thin-layer chromatography of arginine, lysine and ornithine decarboxylase activity among Pseudomonas spp. and Enterobacteriaceae. Microbios 10, 225-231. 